\title{
Abnormal Maturation of Sleep States in Infants with Aborted Sudden Infant Death Syndrome
}

\author{
GABRIEL G. HADDAD, ${ }^{(9)}$ ELLEN M. WALSH, HEDI L. LEISTNER, WARREN K. GRODIN, AND \\ ROBERT B. MELLINS
}

Departments of Pediatrics (Pulmonary Division) and Anesthesiology, Columbia University, College of Physicians and

Surgeons, and Babies Hospital, New York, USA

\begin{abstract}
Summary
The time spent in REM, quiet, and indeterminate sleep was computed in 13 aborted SIDS infants and compared to that spent in the same sleep states in 19 normal infants. Aborted sudden infant death syndrome (SIDS) infants were studied within a week of their aborted SIDS episode and subsequently at monthly intervals through the age of $\mathbf{4}$ months. Normal infants were studied at $1,2,3$, and 4 months of age. Sleep staging was performed by two independent observers using electroencephalogram, electrooculogram, electromyogram and behavioral criteria. Although there was an increase in the percentage of time spent in quiet sleep with age in both normal and aborted SIDS infants, the significantly greater regression coefficients in normal infants $(6.3$ versus $2.9 ; P<0.01)$ indicate that there are differences in the two groups and suggest a maturational abnormality or delay in sleep state distribution in aborted SIDS infants.
\end{abstract}

\section{Speculation}

The results of this study suggest that the differences in sleep state distribution between aborted sudden infant death syndrome and normal infants share a common pathophysiologic alteration with the ventilatory and cardiac abnormalities reported by us elsewhere, perhaps involving the catecholaminergic system.

Previous studies from this laboratory have demonstrated that infants with aborted sudden infant death syndrome (SIDS) have increased heart rate and decreased heart rate variability (7), smaller QT index (3), and a greater $\mathrm{CO}_{2}$-induced increase in minute ventilation (4) than do normal infants during both REM and quiet sleep. We have hypothesized that these findings are most consistent with an increase in the level of sympathoadrenal activity. Because the activity of the autonomic nervous system fluctuates during each sleep state, especially during REM sleep (2), we asked whether these ventilatory and cardiac differences depend on differences in the time spent in each sleep state. We, therefore, compared the time spent in REM, quiet, and indeterminate sleep in 13 aborted SIDS infants and in 19 age-matched normal infants during serial observations at monthly intervals in the first 4 months of life.

\section{MATERIALS AND METHODS}

\section{STUDY POPULATION}

We defined an aborted SIDS infant as one who has experienced at least one episode of unexplained apnea and unresponsiveness with cyanosis or pallor requiring mouth to mouth resuscitation or vigorous physical stimulation for revival. The mean age of the infants at the time of the first aborted SIDS episode was 2 months, with a range of 1 to 4 months. Nine of the 13 infants were observed to be asleep shortly before their apneic episode; the level of consciousness was unknown in the remaining four. Detailed clinical and laboratory assessment failed to reveal an explanation for the aborted SIDS episode. The laboratory evaluation included blood counts, serum glucose, calcium, magnesium, sodium, potassium and chloride, acid-base and blood gas analysis, chest X-ray, conventional 12-lead electrocardiogram, an electroencephalogram (EEG), and a roentgenographic study of the upper gastrointestinal tract following barium swallow.

Ten aborted SIDS infants were full-term, three were preterm and appropriate for gestational age (between 36 and 38 wk gestation). There were five males and eight females. Four had siblings, and two a cousin who died of SIDS. Sleep studies were first performed at about a week after the aborted SIDS episode. Subsequent studies were performed at monthly birthdays through the age of 4 months. Nineteen normal age-matched infants were also studied at 1,2,3, and 4 months of age and served as controls. One infant was preterm ( 37 wk gestation); the rest are full term. There were 12 males and seven males. There was no significant difference in the weights of the two groups either at birth or at any subsequent age studied. Because of imperfect parental compliance and the different ages of presentation of the aborted SIDS infants, the number of normal infants at each age varied from 14 to 17 and that of aborted SIDS infants from 3 to 13 with an average of 16 and 7, respectively. We performed a total of 61 studies in normal and 31 studies in aborted SIDS infants.

All studies were performed after a midmorning feed during natural sleep. This sleep period in our laboratory averaged $2 \frac{1 / 4}{\mathrm{hr}}$ (range, 1 to $3 \mathrm{hr}$ ). Each study was terminated by the natural awakening of the infant, a state characterized by low-voltage, high-frequency EEG, increased bodily movements, and open eyes. No infant was on any medication. In every study, the same techniques and the same laboratory environment were used; the same personnel performed the studies and handled both groups of infants similarly. In addition, because there were no similar studies on aborted SIDS infants, the staff had no a priori notion as to possible differences between the two groups of infants. During each study, as part of a larger project, these infants were also exposed to $2 \% \mathrm{CO}_{2}$ after baseline measurements were made breathing ambient gas concentration.

All subjects are now 6 to 48 months of age and are neurologically normal. Informed written consent was obtained from the parents of all infants.

\section{SLEEPING STAGING}

Standard skin surface electrodes were used to record three neurophysiologic signals: a C4-A1 EEG, a bipolar electrooculogram, and bipolar submental electromyogram. The resistance of these electrodes was less than $10,000 \mathrm{ohms}$, and the signals were noise free. A behavioral code (8) that described the infant's somatic activities during sleep was recorded throughout each study by an observer. Using the EEG, electrooculogram, and electromyogram and behavioral criteria, sleep was staged visually on 30- 
sec epochs (1) by two independent observers. In over 85 to $90 \%$ of instances, both observers agreed on the sleep state. In the remaining periods, a decision was reached after careful rereview of the sleep records.

\section{STATISTICAL ANALYSIS}

Regression analysis was performed on all the individual data to characterize the maturation of the sleep pattern in both normal and aborted SIDS infants. Significant differences were assessed by the Student $t$ test for unmatched samples. Differences were considered significant when $P$ values were less than 0.05 .

\section{RESULTS}

Because there were no significant differences in the distribution of sleep states when infants were breathing ambient gas concentrations and when they were breathing $2 \% \mathrm{CO}_{2}(P>0.1)$, all data for each sleep state have been pooled for purposes of data analysis. Linear regression analysis reveals a significant increase with age in the time spent in quiet sleep (Fig. 1) and a significant decrease in the time spent in REM sleep (Fig. 3) in both normal and aborted SIDS infants $(P<0.05)$. The regression coefficients are greater in normal than in aborted SIDS infants when the percentage of sleep time is analyzed as a function of age in both REM $(-7.0$ versus -5.7$)$ and quiet sleep $(6.3$ versus 2.9$)$, but this is significant only in quiet sleep $(P<0.01)$. There is no significant change in the time spent in indeterminate sleep with age for normal infants, but there is a small but significant increase in the time spent in indeterminate sleep with age for aborted SIDS infants $(P<0.05)$ (Fig. 2). There is no significant difference between females and males in each group at each age $(P>0.01)$. There is no significant difference in the total length of sleep at any age between normal and aborted SIDS infants $(P>0.1)$.

\section{DISCUSSION}

The change in percent time in REM, quiet, and indeterminate sleep with age in our normal infants is consistent with the findings of others (8). The present work shows that the decrease in REM

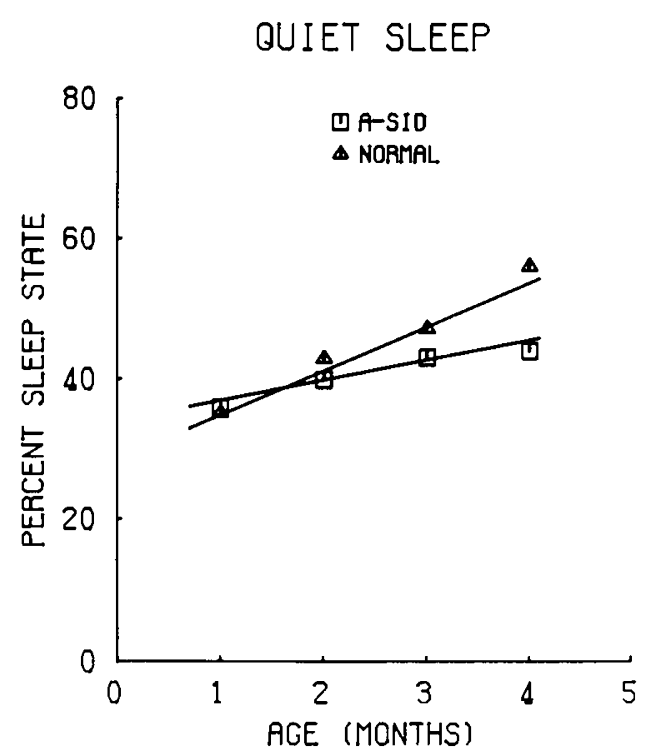

Fig. 1. The increase in the percent quiet sleep (time spent in quiet sleep divided by total sleep time) as a function of age is significantly greater in normal than in aborted SIDS infants. Linear regression equations for the plotted lines are as follows: for normal infants, $y=6.3 x+28.55$; for aborted SIDS infants, $y=2.9 x+34.06$. Plotted lines represent regression functions based on all individual data points. Means of individual data points are shown at each age. See text.
INDETERMINATE SLEEP

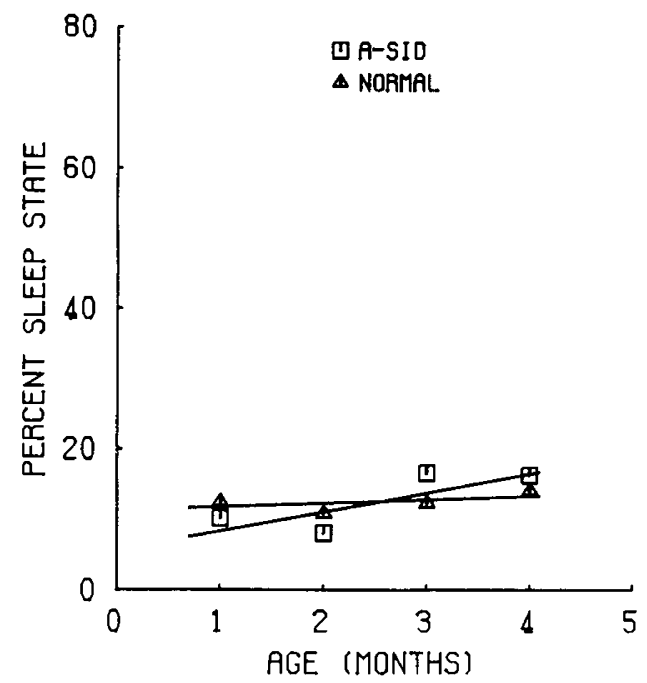

Fig. 2. Aborted SIDS infants and normal infants spend approximately the same fraction of their sleep in indeterminate sleep at all ages. Linear regression equations for the plotted lines are as follows: for normal infants, $y=0.51 x+11.3$; for aborted SIDS infants, $y=2.7 x+5.66$. Plotted lines represent regression functions based on all individual points. Means of individual data points are shown at each age. See text.

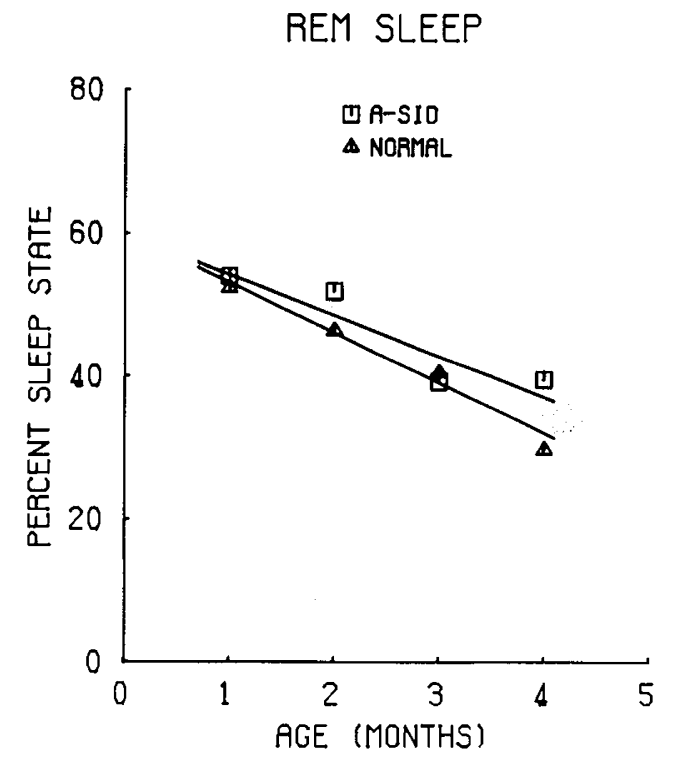

Fig. 3. The decrease in the percent REM (time spent in REM sleep divided by total sleep time) as a function of age is greater in normal than in aborted SIDS infants. Linear regression equations for the plotted lines are as follows: for normal infants, $y=-7.0 x+60.15$; for aborted SIDS infants, $y=-5.7 x+59.96$. Plotted lines represent regression functions based on all individual points. Means of individual points are shown at each age. See text.

and increase in quiet sleep with age is also present in aborted SIDS infants. However, although the direction of change with age in the percent time in REM and quiet sleep is the same for the two groups of infants, the magnitude of this change is greater in normal infants, especially in quiet sleep. Also, our aborted SIDS infants, unlike normal infants (8), have a small increase in indeterminate sleep with age. Hence, the distribution of sleep states is abnormal, and the maturation of this distribution is possibly delayed in aborted SIDS infants when compared to normal infants. Although the relationship of our findings to SIDS is not clear, it is of interest to note that the differences in sleep state 
distribution between normal and aborted SIDS infants do not appear, especially in quiet sleep, until after 1 month of age and are present during the period when most cases of SIDS occur, namely between 2 and 4 months.

Although we have shown differences in sleep state distribution between both groups of infants, this work has some limitation because each sleep study was limited to an average of $2 \frac{1 / 4}{4 h}$. However, we have also seen in the same studies ventilatory and cardiac differences between aborted SIDS and normal infants in both REM and quiet sleep $(3,4,7)$. Therefore, in contrast to the difference in the distribution of sleep states occurring with age over the first 4 months of life, these ventilatory and cardiac differences did not change with age. Hence, we believe that it is unlikely that the cardiac and ventilatory differences between these two groups of infants are the result of differences in the percent time spent in each sleep state.

The basis for the abnormal maturation in the distribution of sleep states in the aborted SIDS infants is not clear. There is, however, evidence in animals that central levels of catecholamines modulate sleep state distribution $(5,6)$. We have previously hypothesized that aborted SIDS have an increased in sympathoadrenal activity $(3,4,7)$. Whether the cardiac and ventilatory abnormalities and the differences in sleep state distribution in aborted SIDS infants share a common pathophysiologic alteration perhaps involving catecholamines is not known but may be worth pursuing to further our understanding of the pathogenesis of SIDS.

\section{REFERENCES AND NOTES}

1. Anders, T., Emde, R., and Parmelee, A.: A Manual of Standardized Terminology, Techniques and Criteria for Scoring of States of Sleep and Wakefulness in Newborn Infants. (BRI Publication, Los Angeles, 1971).

2. Baust, W., and Bohmert, B.: The regulation of heart rate during sleep. Exp. Brain Res., 7: 169 (1969).

3. Haddad, G. G., Epstein, M. A. F., Epstein, R. A., Mazza, N. M., Mellins, R. B., and Krongrad, E.: The QT interval in aborted SIDS infants. Pediatr. Res., 13: 136 (1979).

4. Haddad, G. G., Leistner, H. L., Epstein, R. A., Epstein, M. A. F., Melnikoff, B., and Mellins, R. B.: Abnormal breathing pattern and increased ventilatory response to $\mathrm{CO}_{2}$ in aborted SIDS infants during sleep. Am. Rev. Respir. Dis. (Abstract), 119: 267 (1979).

5. Holman, R. B., Elliott, G. R., and Barchas, J. D.: Neuroregulators and sleep mechanisms. Ann. Rev. Intern. Med., 26: 499 (1975).

6. Jouvet, M.: The regulation of sleep/waking cycle by monoaminergic neurons in the cat. In: H. M. Van Praag, H. Meinardi: Brain and Sleep. p. 22 (De Erven Bohn BV, Amsterdam, 1974).

7. Leistner, H. L., Haddad, G. G., Epstein, R. A., Lai, L. T., Epstein, M. A. F., and Mellins, R. B.: Heart rate and heart rate variability during sleep in aborted sudden infant death syndrome. J. Pediatr., 97: 51 (1980).

8. Parmelee, A. H., and Stern E.: Development of states in infants. In: C. B. Clemente, D. P. Purpura, F. E. Mayer: Sleep and the Maturing Nervous System. p. 199 (Academic Press, Inc., New York, 1972).

9. Requests for reprints should be addressed to: G. G. Haddad, M. D., Pediatric Pulmonary Division, Columbia-Presbyterian Medical Center, 622 West 168th Street, New York, NY 10032 (USA).

10. This research was supported by National Institutes of Health Grants HD 08297 , HL 07421, RR 00645, Contract 52856 and a grant from the Parker B. Francis Foundation.

11. Received for publication March 18, 1980.

12. Accepted for publication November 12, 1980. 